\title{
Niclosamide suppresses migration of hepatocellular carcinoma cells and downregulates matrix metalloproteinase-9 expression
}

\author{
MINORU TOMIZAWA ${ }^{1}$, FUMINOBU SHINOZAKI ${ }^{2}$, YASUFUMI MOTOYOSHI ${ }^{3}$, \\ TAKAO SUGIYAMA $^{4}$, SHIGENORI YAMAMOTO ${ }^{5}$ and NAOKI ISHIGE ${ }^{6}$ \\ Departments of ${ }^{1}$ Gastroenterology, ${ }^{2}$ Radiology, ${ }^{3}$ Neurology, ${ }^{4}$ Rheumatology, ${ }^{5}$ Pediatrics and ${ }^{6}$ Neurosurgery, \\ National Hospital Organization Shimoshizu Hospital, Yotsukaido, Chiba 284-0003, Japan
}

Received August 31, 2014; Accepted May 21, 2015

DOI: $10.3892 / \mathrm{ol} .2015 .3789$

\begin{abstract}
Metastasis negatively affects the prognosis of hepatocellular carcinoma (HCC). In the present study, niclosamide, which is known to suppress the proliferation of HCC cells, was investigated for possible suppressant effects on the migration of HCC cells. HLF and PLC/PRF/5 HCC cells were cultured in the presence of niclosamide. Cell proliferation was analyzed using the MTS assay. Cell migration was measured by performing a scratch assay. Expression levels of cyclin D1 and matrix metalloproteinase 9 (MMP9) were analyzed by performing revers transcription-quantitative polymerase chain reaction. Compared with the control treatment, treatment with $10 \mu \mathrm{m}$ niclosamide suppressed the proliferation of the HLF and PRL/PRF/5 cells to $49.9 \pm 3.7$ and $17.9 \pm 11.5 \%(\mathrm{P}<0.05)$, respectively. Furthermore, compared with the control treatment, treatment with $1.0 \mu \mathrm{M}$ niclosamide downregulated the expression of cyclin D1 to $52.4 \pm 4.4$ and $23.9 \pm 5.4 \%(\mathrm{P}<0.05)$ in the HLF and PRL/PRF/5 cells, respectively. In the scratch assay, treatment of the HLF cells with niclosamide $(1.0 \mu \mathrm{m})$ decreased the distance of the scratched line from the growing edge to $4.6 \pm 1.0 \mathrm{~mm}$ compared with the $9.2 \pm 1.4 \mathrm{~mm}$ observed with the control treatment $(\mathrm{P}<0.05)$. Similarly, treatment of the PRL/PRF/5 cells with niclosamide $(1.0 \mu \mathrm{m})$ also decreased the distance of the scratched line from the growing edge to $3.0 \pm 0.8 \mathrm{~mm}$ compared with the $5.5 \pm 0.9 \mathrm{~mm}$ observed with the control treatment $(\mathrm{P}<0.05)$. Further, MMP9 expression levels in the HLF cells treated with $1.0 \mu \mathrm{m}$ niclosamide decreased to $22.4 \pm 1.76 \%(\mathrm{P}<0.05)$ compared with those in the untreated control HLF cells. Similarly, expression level of MMP9 in the PRL/PRF/5 cells treated with $1.0 \mu \mathrm{m}$ niclosamide deceased to $18.7 \pm 10.7 \%$ $(\mathrm{P}<0.05)$ compared with those in the untreated control PRL/
\end{abstract}

Correspondence to: Dr Minoru Tomizawa, Department of Gastroenterology, National Hospital Organization Shimoshizu Hospital, 934-5 Shikawatashi, Yotsukaido, Chiba 284-0003, Japan E-mail: nihminor-cib@umin.ac.jp

Key words: scratch assay, quantitative polymerase chain reaction, MTS assay
PRF/5 cells. Overall, niclosamide downregulated the expression of MMP9 in and suppressed the migration of HCC cells.

\section{Introduction}

Hepatocellular carcinoma (HCC) is one of the most common malignancies and its prognosis is poor with metastasis $(1,2)$. HCC cells migrate at an early stage (3). One of the problems associated with $\mathrm{HCC}$ is that its cells migrate into the surrounding fibrous tissues and proliferate (4). In vitro, HCC cells can proliferate without nutrients or growth factors such as fetal bovine serum (FBS) (5). It is important to detect molecular events at an early stage of HCC cell migration to improve HCC treatment. Matrix metalloproteinase 9 (MMP9) expression level is upregulated 20 -fold after co-culture of HCC cells and parenchymal cells (6).

MMP9 is a gelatinase involved in cancer metastasis (7). The overexpression of MMP9 is associated with the poor prognosis of cancer (8). Research focused on elucidating the mechanisms involved in the upregulation of MMP9 expression is therefore important. Polymorphism of the promoter region of MMP9 (-1562)C/T increases the risk of metastasis (9). MMP9 is upregulated in the presence of inflammatory cytokines such as interleukins 8 and 17 (10). However, details of the mechanisms involved in the upregulation of MMP9 expression in HCC are not clear.

The Wnt signaling pathway, which is activated when Wnt ligands bind their Frizzled (Fz) receptors, has been implicated in HCC (11). The small interfering (si)RNA of Fz9 suppresses the proliferation and migration of HCC cells (12). Studies suggest that the Wnt pathway is specifically involved in the migration of HCC cells, and it is expected that inhibition of this pathway may improve the survival rate of HCC patients by suppressing the proliferation and migration of HCC cells. One limitation of siRNA, however, is that its antitumor effect depends on its transfection efficiency (13).

Niclosamide was originally developed for the treatment of tapeworm infection and is clinically used worldwide $(14,15)$. Sack et al first suggested that niclosamide may be a promising candidate for the treatment of colorectal cancer through the inhibition of HCC cell proliferation (16) via the modulation of the Wnt pathway (17). The mechanisms proposed for the Wnt pathway-mediated inhibitory effects of 
niclosamide include internalization of Fz1 (18) and inhibition of the canonical Wnt signaling pathway (19). Niclosamide also downregulates the expression of cyclin D1, one of the target molecules of the Wnt pathway, via glycogen synthase kinase- $3 \beta(17,20)$. Thus far, no study has reported the effects of niclosamide on the migration of HCC cells or on its regulation of MMP9 expression.

The present study was therefore conducted to investigate the effects of niclosamide on the migration of HCC cells and the expression of MMP9.

\section{Materials and methods}

Cell culture. HCC HLF and PLC/PRL/5 cell lines were purchased from Riken Cell Bank (Tsukuba, Japan). The cells were cultured in Dulbecco's modified Eagle's medium (DMEM) (Sigma-Aldrich, St. Louis, MO, USA) supplemented with 10\% FBS (Life Technologies, Grand Island, NY, USA) and incubated in a humidified chamber at $37^{\circ} \mathrm{C}$ in $5 \%$ carbon dioxide.

Scratch assay. The cells were plated on 4-well chamber slides (BD Biosciences, Franklin Lakes, NJ, USA) and allowed to yield a confluent monolayer sheet of cells, which were then scratched with $200-\mu \mathrm{l}$ pipettes, incubated for $48 \mathrm{~h}$ and stained with hematoxylin and eosin. The stained slides were observed under an AX80 microscope (Olympus, Tokyo, Japan). The distance from the location of the original scratched line to the new growing edge of the cells was measured at five different points. The migration distance for each plate was calculated as the mean average of these five measurements.

Cell proliferation analysis. The HLF and PRL/PRF/5 cells were trypsinized, harvested, seeded onto 96-well flat-bottomed plates (Asahi Techno Glass, Tokyo, Japan) at a density of 1,000 cells per well and then incubated for $24 \mathrm{~h}$ in DMEM supplemented with 10\% FBS. Subsequent to culturing, the cells were treated with niclosamide (Sigma-Aldrich) at various concentrations $(0,0.01,0.03,0.1$, $0.3,1,3$ and $10 \mu \mathrm{M}$ ) for $72 \mathrm{~h}$ and subjected to MTS assay (Promega Corporation, Madison, WI, USA) according to the manufacturer's instructions. MTS is bio-reduced by cells into a colored formazan product that can be detected at a specific wavelength. The absorbance of each reaction plate was measured at a wavelength of $490 \mathrm{~nm}$ using an iMark Microplate Absorbance Reader (Bio-Rad Laboratories Inc., Hercules, CA, USA).

Reverse transcription-quantitative polymerase chain reaction (RT-qPCR). The cells were cultured in 6-well plates (Asahi Glass Co., Ltd., Tokyo, Japan) and treated with niclosamide for $48 \mathrm{~h}$, after which total RNA (5 $\mu \mathrm{g})$ was isolated using Isogen (Nippon Gene Co.,Ltd., Tokyo, Japan). The RNA was converted reverse transcribed into cDNA using the Super Script III and oligo(dT) primers (Life Technologies Ltd., Carlsbad, CA, USA) according to the manufacturer's instructions. The PCR primers were as follows: Ribosomal protein L19 (RPL19; BC095445), forward 5'-CGAATGCCAGAGAAGGTCAC-3' and reverse 5'-CCATGAGAATCCGCTTGTTT-3' (157 bp); cyclin D1 (NM_053056), forward 5'-AGAGGCGGAGGAGAACA AACAG-3' and reverse 5'-AGGCGGTAGTAGGACAG
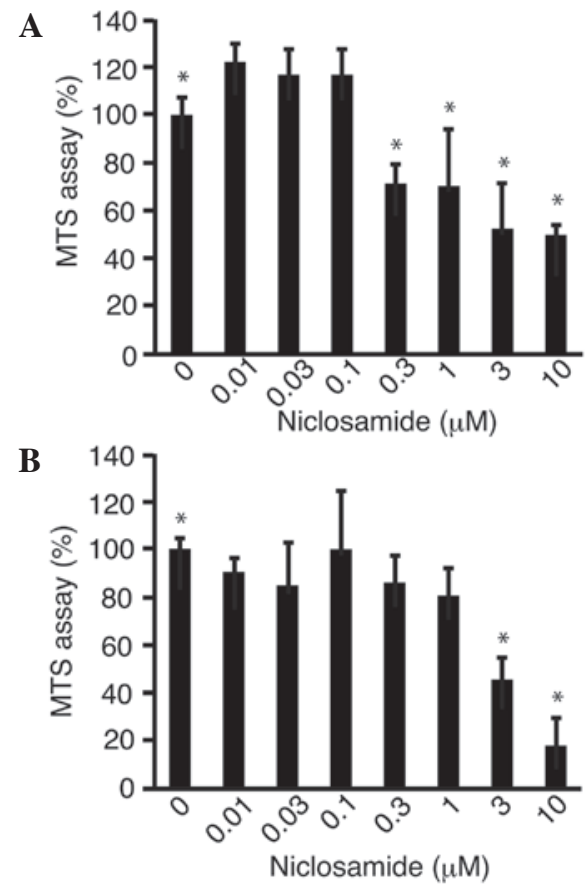

Figure 1. Cell proliferation assay. (A) HLF cells or (B) PLC/PRL/5 cells were cultured with niclosamide and subjected to MTS assays. The error bars represent the standard deviation $(\mathrm{n}=3)$. ${ }^{*} \mathrm{P}<0.05$ vs. $0 \mu \mathrm{M}$.

A

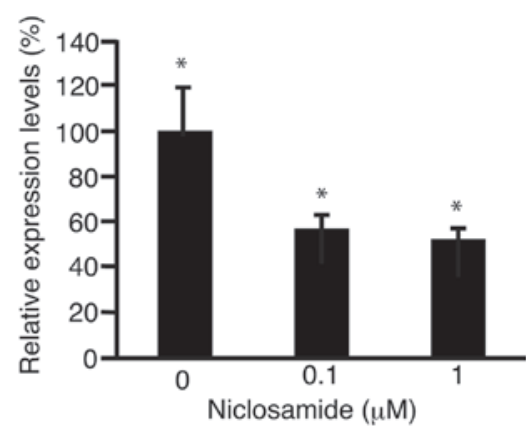

B

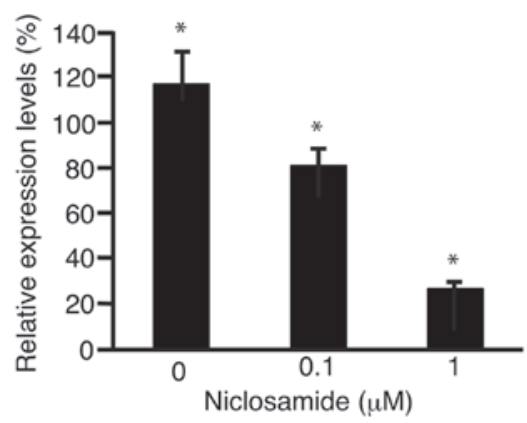

Figure 2. Reverse transcription-quantitative polymerase chain reaction (RT-qPCR) of cyclin D1. (A) HLF cells or (B) PLC/PRL/5 cells were cultured with niclosamide, and subjected to qRT-PCR for detection of cyclin D1 expression. The error bars represent the standard deviation $(\mathrm{n}=3)$. ${ }^{*} \mathrm{P}<0.05$ vs. $0 \mu \mathrm{M}$.

GAAGTTG-3' (180 bp); and MMP-9 (NM_004994) forward 5'-CCTGGGCAGATTCCAAACCT-3' and reverse 5'-GCAAGTCTTCCGAGTAGTTTTGGAT-3' (89 bp). RT-qPCR was performed using Fast SYBR Green Master Mix (Life Technologies Ltd.) for 40 cycles, using $5 \mathrm{sec}$ for denaturation at $95^{\circ} \mathrm{C}$ and $5 \mathrm{sec}$ for annealing-extension at $60^{\circ} \mathrm{C}$ with 

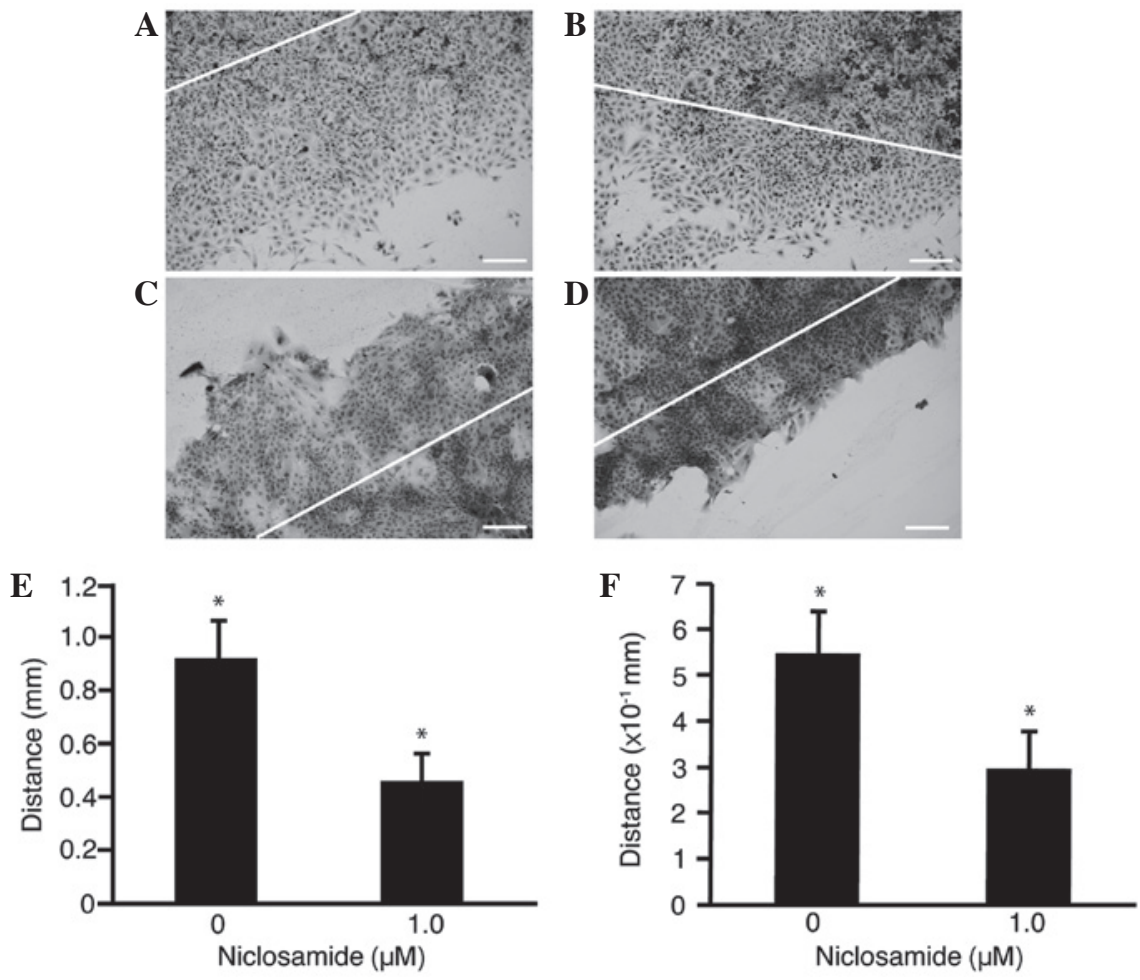

Figure 3. Scratch assay. (A and B) HLF or (C and D) PLC/PRL/5 cells were cultured on chamber slides. Upon attaining confluence, the cells were scratched with the tip of a 200- $\mu$ l pipette tip and treated (B and D) with $1.0 \mu \mathrm{M}$ niclosamide, or (A and C) without niclosamide. The white line represents the location of the original leading edge of the scratch. The distance from the line to the new growing edge of the cells was measured in the (E) HLF and (F) PLC/PRL/5 cells. Original magnification, $\mathrm{x} 100$; scale bar, $200 \mu \mathrm{m}$; error bar, standard deviation; $\mathrm{n}=3$; ${ }^{*} \mathrm{P}<0.05$ vs. $0 \mu \mathrm{M}$.

the Mini Opticon System (Bio Rad, Hercules, CA). RPL19 was used as an internal control.

Statistical analysis. One-factor analysis of variance was performed on the data from the cell proliferation studies, RT-qPCR and scratch assays using JMP10.0.2 software (SAS Institute, Cary, NC, USA). $\mathrm{P}<0.05$ was used to indicate a statistically significant difference.

\section{Results}

The effects of niclosamide on the proliferation of the HLF (Fig. 1A) and PRL/PRF/5 (Fig. 1B) cells were examined using the MTS assay. The proliferation of the HLF and PRL/PRF/5 cells treated with niclosamide $(10 \mu \mathrm{m})$ was significantly $(\mathrm{P}<0.05)$ suppressed to $49.9 \pm 3.7$ and $17.9 \pm 11.5 \%$, respectively, compared with that of the untreated control cells.

Cyclin D1 is a downstream molecule associated with the Wnt pathway and involved in cell proliferation (21). The HLF and PRL/PRF/5 cells were cultured with niclosamide. The expression levels of cyclin D1 in the HLF (Fig. 2A) and PRL/PRF/5 (Fig. 2B) cells cultured in the presence of niclosamide were analyzed using RT-qPCR. The results showed that cyclin D1 expression was significantly $(\mathrm{P}<0.05)$ downregulated to $52.4 \pm 4.4$ and $23.9 \pm 5.4 \%$ in the HLF and $\mathrm{PRL} / \mathrm{PRF} / 5$ cells, respectively, compared with the levels in the untreated control cells.

Change in cell motility was also investigated in the HLF (Fig. 3A and B) and PRL/PRF/5 (Fig. 3C and D) cells treated with niclosamide $(1.0 \mu \mathrm{m})$. A reduction in the distance of the
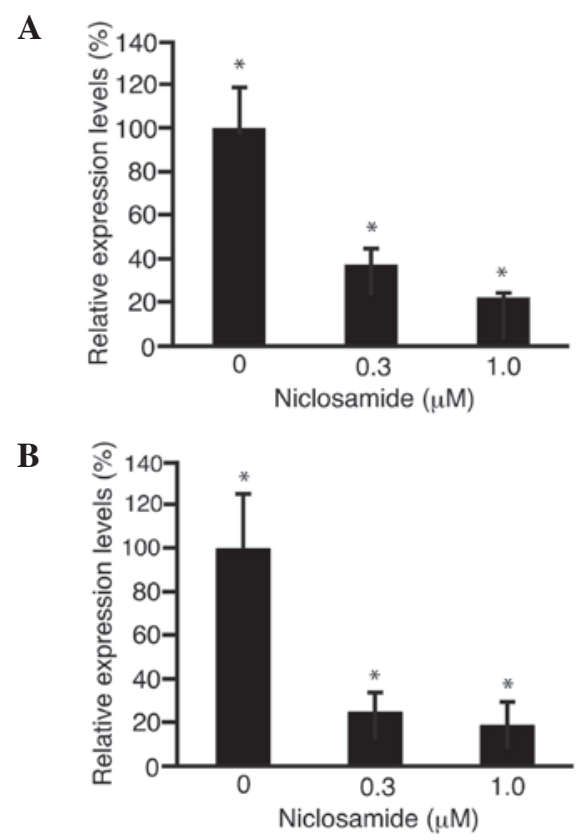

Figure 4. Real-time quantitation polymerase chain reaction (qRT-PCR) of matrix metalloproteinase 9. HLF cells (A) and PLC/PRL/5 cells (B) were cultured with niclosamide and subjected to qRT-PCR with matrix metalloproteinase 9. Error bar: standard deviation, ${ }^{*} \mathrm{P}<0.05$ vs. $0 \mu \mathrm{M}, \mathrm{n}=3$.

scratched line from the growing edge was observed in the treated cells (Fig. 3B and D) compared with the untreated control cells (Fig. 3A and C). The HLF (Fig. 3E) and PRL/PRF/5 (Fig. 3F) cells treated with niclosamide showed significant $(\mathrm{P}<0.05)$ decreases in cell migration $(4.6 \pm 1.0$ and 
$3.0 \pm 0.8 \mathrm{~mm}$, respectively) compared with that of the untreated control HLF and PRL/PRF/5 cells $(9.2 \pm 1.4$ and $5.5 \pm 0.9 \mathrm{~mm}$, respectively).

MMP9 is involved in cell motility, and as expected, its expression level in the HLF (Fig. 4A) and PRL/PRF/5 (Fig. 4B) cells treated with niclosamide $(1.0 \mu \mathrm{m})$ was significantly $(\mathrm{P}<0.05)$ decreased to $22.4 \pm 1.76 \%$ and $18.7 \pm 10.7 \%$ compared with that in the untreated cells.

\section{Discussion}

Niclosamide is currently used to treat tapeworm infection (15). Application of niclosamide in the treatment of cancer was first reported for colorectal cancer (16). Niclosamide suppresses the proliferation of HCC cells by inhibiting the Wnt signaling pathway (17).

Niclosamide has been previously shown to suppress the migration of breast cancer cells and colon cancer cells $(16,22)$. The present results revealed a similar suppression of HCC cell migration by niclosamide, suggesting that it may suppress the migration of cancer cells. These results are promising and may be clinically significant, as niclosamide could improve the prognosis of cancer patients by suppressing metastasis. While the mechanism by which niclosamide suppresses the migration of cancer cells was not completely clear, the present study clearly showed that MMP9 expression was downregulated by niclosamide, thereby strongly suggesting its involvement in the suppression of cancer cell migration. Previous studies and the present study have indicated that niclosamide is promising for the treatment of HCC (23).

The present data clearly showed that niclosamide suppressed the migration of HCC cells, and that MMP9 decreased with niclosamide treatment. This suggests that niclosamide suppresses migration via inhibiting the expression of MMP9 (7). However, the mechanism underlying decreased MMP9 expression remains unclear. Our previous study revealed that niclosamide suppresses the Wnt pathway (17). Therefore, it is suggested that the expression of MMP9 decreases with suppression of the Wnt pathway, and that MMP9 may be a downstream molecule of the Wnt pathway.

In conclusion, niclosamide significantly suppressed the migration of the HCC cells, probably by mechanisms involving the downregulation of MMP9 expression. Future studies in line with these results could involve investigation of the promoter activity of MMP9, which would further elucidate the effects of niclosamide at a transcriptional level.

\section{References}

1. Tabrizian P, Roayaie S and Schwartz ME: Current management of hepatocellular carcinoma. World J Gastroenterol 20: 10223-10237, 2014.

2. Wang $\mathrm{H}$ and Chen L: Tumor microenviroment and hepatocellular carcinoma metastasis. J Gastroenterol Hepatol 28 (Suppl 1): S43-S48, 2013

3. Kondo F, Kondo Y, Nagato Y, Tomizawa M and Wada K: Interstitial tumour cell invasion in small hepatocellular carcinoma. Evaluation in microscopic and low magnification views. J Gastroenterol Hepatol 9: 604-612, 1994.
4. Tomizawa M, Kondo F and Kondo Y: Growth patterns and interstitial invasion of small hepatocellular carcinoma. Pathol Int 45: 352-358, 1995.

5. Tomizawa M, Shinozaki F, Sugiyama T, Yamamoto S, Sueishi M and Yoshida T: Sorafenib suppresses the cell cycle and induces the apoptosis of hepatocellular carcinoma cell lines in serum-free media. Exp Ther Med 1: 863-866, 2010.

6. Chen RX, Song HY, Dong YY, Hu C, Zheng QD, Xue TC, Liu XH, Zhang Y, Chen J, Ren ZG, et al: Dynamic expression patterns of differential proteins during early invasion of hepatocellular carcinoma. PLoS One 9: e88543, 2014.

7. Vandooren J, Van den Steen PE and Opdenakker G: Biochemistry and molecular biology of gelatinase B or matrix metalloproteinase-9 (MMP-9): The next decade. Crit Rev Biochem Mol Biol 48: 222-272, 2013.

8. Li H, Zhang K, Liu LH, Ouyang Y, Bu J, Guo HB and Xiao T: A systematic review of matrix metalloproteinase 9 as a biomarker of survival in patients with osteosarcoma. Tumour Biol 35: 5487-5491, 2014.

9. Liu D, Guo H, Li Y, Xu X, Yang K and Bai Y: Association between polymorphisms in the promoter regions of matrix metalloproteinases (MMPs) and risk of cancer metastasis: A meta-analysis. PLoS One 7: e31251, 2012.

10. Zarogoulidis P, Katsikogianni F, Tsiouda T, Sakkas A, Katsikogiannis $\mathrm{N}$ and Zarogoulidis K: Interleukin-8 and interleukin-17 for cancer. Cancer Invest 32: 197-205, 2014.

11. Pez F, Lopez A, Kim M, Wands JR, Caron de Fromentel C and Merle P: Wnt signaling and hepatocarcinogenesis: Molecular targets for the development of innovative anticancer drugs. J Hepatol 59: 1107-1117, 2013.

12. Fujimoto T, Tomizawa M and Yokosuka O: SiRNA of frizzled-9 suppresses proliferation and motility of hepatoma cells. Int $\mathrm{J}$ Oncol 35: 861-866, 2009.

13. Wang YQ, Wang F, Deng XQ, Sheng J, Chen SY and Su J: Delivery of therapeutic AGT shRNA by PEG-Bu for hypertension therapy. PLoS One 8: e68651, 2013.

14. Knorr R: Treatment of tapeworm with Yomesan in 36 patients. Med Lav 55: 1937-1938, 1960 (In German).

15. Mwape KE, Phiri IK, Praet N, Muma JB, Zulu G, Van den Bossche P, de Deken R, Speybroeck N, Dorny P and Gabriël S: Taenia solium Infections in a rural area of Eastern Zambia-a community based study. PLoS Negl Trop Dis 6: e1594, 2012.

16. Sack U, Walther W, Scudiero D, Selby M, Kobelt D, Lemm M, Fichtner I, Schlag PM, Shoemaker RH and Stein U: Novel effect of antihelminthic Niclosamide on S100A4-mediated metastatic progression in colon cancer. J Natl Cancer Inst 103: 1018-1036, 2011.

17. Tomizawa M, Shinozaki F, Motoyoshi Y, Sugiyama T, Yamamoto S, Sueishi M and Yoshida T: Niclosamide suppresses Hepatoma cell proliferation via the Wnt pathway. Onco Targets Ther 6: 1685-1693, 2013.

18. Chen M, Wang J, Lu J, Bond MC, Ren XR, Lyerly HK, Barak LS and Chen W: The anti-helminthic niclosamide inhibits Wnt/ Frizzled1 signaling. Biochemistry 48: 10267-10274, 2009.

19. Wieland A, Trageser D, Gogolok S, Reinartz R, Höfer H, Keller M, Leinhaas A, Schelle R, Normann S, Klaas L, et al: Anticancer effects of niclosamide in human glioblastoma. Clin Cancer Res 19: 4124-4136, 2013.

20. Takahashi-Yanaga F and Sasaguri T: GSK-3beta regulates cyclin D1 expression: A new target for chemotherapy. Cell Signal 20: 581-589, 2008.

21. Takayasu H, Horie H, Hiyama E Matsunaga T, Hayashi Y, Watanabe Y, Suita S, Kaneko M, Sasaki F, Hashizume K, et al: Frequent deletions and mutations of the beta-catenin gene are associated with overexpression of cyclin D1 and fibronectin and poorly differentiated histology in childhood hepatoblastoma. Clin Cancer Res 7: 901-908, 2001.

22. Ye T, Xiong Y, Yan Y, Xia Y, Song X, Liu L, Li D, Wang N, Zhang L, Zhu Y, et al: The anthelmintic drug niclosamide induces apoptosis, impairs metastasis and reduces immunosuppressive cells in breast cancer model. PLoS One 9: e85887, 2014.

23. Li Y, Li PK, Roberts MJ, Arend RC, Samant RS and Buchsbaum DJ: Multi-targeted therapy of cancer by niclosamide: A new application for an old drug. Cancer Lett 349: 8-14, 2014. 